\title{
EL CUIDADO PROFESIONAL DE ENFERMERÍA UN ENFOQUE HUMANÍSTICO
}

\section{PROFESSIONAL NURSING CARE HUMANÍSTICO APPROACH}

\author{
Hinostroza Robles, Nelly Marleni ${ }^{1, a, b, c}$
}

${ }^{1}$ Universidad Nacional Daniel Alcides Carrión - Cerro de Pasco.

${ }^{a}$ Licenciada en Enfermería. ${ }^{b}$ Especialista en investigación en Ciencias de la Salud y Psicología, ${ }^{c}$ Magister en Enfermería.

\section{RESUMEN:}

Objetivos: Identificar, analizar e interpretar las investigaciones relacionadas al cuidado de enfermería desde un enfoque humanístico en los servicios hospitalarios en Latinoamérica y socializar el marco teórico filosófico de J. Phaterson, J. Watson y E. Wiedenbach. Método: Descriptivo-comparativo de investigaciones cualitativas y cuantitativas de Cuidado Humanístico en Enfermería. Se realizaron cuarenta revisiones científicas relacionados al tema y se seleccionaron diez por su especificidad temática y contextual. Hallazgos: Las investigaciones realizadas en Latinoamérica, demuestran que hay factores sociales y profesionales que influyen en el cuidado humanizado, los sistemas complejos de salud en las instituciones públicas, la escasez de cursos humanos y materiales y la influencia del paradigma tecno científico limitan el ejercicio de la ciencia y arte de enfermería, de un encuentro genuino con el paciente para explorar sus vivencias durante la enfermedad y cuidarlo. Consideraciones Finales: El cuidado de enfermería desde un enfoque humanístico, sugiere al profesional de enfermería dar una mirada filosófica de la persona, en una relación existencial, transaccional y una conciencia intelectual que permita la identidad y empoderamiento profesional de enfermería.

Palabras claves: Cuidado profesional, enfermería humanística, existencialismo, fenomenología.

\section{SUMMARY:}

Objectives: To identify, analyze and interpret research related to nursing care from a humanistic approach in hospital services in Latin America and socialize the philosophical framework of Paterson J., J. Watson and E. Wiedenbach. Method: Descriptive - comparative qualitative and quantitative research of Humanistic Nursing Care. Forty scientific reviews related to the topic and selected ten themes and their contextual specificity were performed. Findings: Research in Latin America show that there are social and occupational factors influencing the humanized care, complex health systems in public institutions, the shortage of human and material courses and the influence of scientific technological paradigm limit the exercise science art and nursing, a genuine encounter with the patient to explore their experiences during the illness and care. Final Thoughts: The nursing care from a humanistic approach suggests the nurse give a philosophical view of the person, in an existential, transactional relationship and intellectual consciousness that allows the identity and professional empowerment of nursing.

Keywords: Professional care, humanistic nursing, existentialism, phenomenology. 


\section{INTRODUCCIÓN}

Mi interés de realizar este artículo de revisión científica es la promoción de un diálogo de enfermería y el compromiso con la práctica sustentado en la teoría humanista, ésta permitirá un cuidado de calidad al individuo, familia y comunidad durante el proceso salud- enfermedad y el cumplimiento del deber esto es, brindar atención eminentemente humana, oportuna, continua y segura, considerando la individualidad de la persona a quien cuida. (1)

Cuando hablamos de cuidado profesional en enfermería, debemos asumir que esta se da en una relación de enfermera-paciente, con esmero y atención (2), afecto, preocupación y responsabilidad valores que ayudan a crecer a ambos (3) y que es un elemento paradigmático que diferencia a la enfermería de otras profesiones del área de la salud, considerada como la más bella de las profesiones; porque en el cuidado se descubre la expresión de la humanidad y reflexión de nuestros valores, principios profesionales y personales y se puede describir el cuidado profesional como un rasgo humano, como un imperativo moral, como afecto, como un proceso de interacción personal y como intervención terapéutica (4), además de considerar en el proceso interactivo la dimensión estética y ética. Todos estos componentes tienen un fundamento filosófico que sustentan científicamente la práctica de enfermería, descritos por Jean Watson, Josephine Paterson y Ernestine Wiedenbach basados en el existencialismo y la fenomenología y asumir el paradigma de la transformación del cuidado $(5,6,7,8)$.

Considerando estas premisas, se observa que existe una paradoja en la práctica: el modelo biomédico se hace predominante, existiendo escasa relación con el paciente de allí que surgen las consideraciones de deshumanización del cuidado. Se hace necesario indagar las evidencias científicas de enfermería desde un enfoque humanístico para no descuidar la esencia profesional: "EI cuidado humano". Mediante la tabla sugerida por Burns y Grove (9) se identificaron evidencias científicas de la última década en Latinoamérica relacionadas al Cuidado Humanístico de Enfermería. También se debe recordar la base teórica que sustenta el actuar de la enfermería como ciencia y como arte. Desde el punto de vista filosófico Enfermería exige un compromiso categórico en la búsqueda de conocimientos que promuevan el desarrollo profesional para el beneficio de la humanidad (10). Las diez investigaciones clasificadas $(11,12,13,14$, $15,16,17,18,19,20)$ corresponden a contextos hospitalarios. Los resultados evidencian cómo se está ejerciendo la enfermería, que en los últimos años se ha debilitado y ha perdido espacios sociales merecidamente ganados en países desarrollados. Esto permitirá una reflexión del ejercicio de la enfermería en nuestro país y mostrar evidencia de nuestro actuar.

El objetivo fue identificar, analizar e interpretar las investigaciones relacionadas al cuidado de enfermería desde un enfoque humanístico en los servicios hospitalarios en Latinoamérica y socializar el marco teórico filosófico de J. Phaterson, J. Watson y E. Wiedenbach.

\section{METODOLOGÍA}

Se hizo una revisión bibliográfica de varias fuentes, a través de los descriptores en CUIDEN PLUS, DOGPILE, PUBMED, LILACS, ridec. Los registros de las investigaciones oscilaron entre cuarenta, seleccionando diez revisiones científicas, relacionadas específicamente al cuidado humanístico de enfermería en el contexto hospitalario.

Las revisiones científicas se describieron y compararon según el esquema de Burns y Grove (Ver tabla 1, 2, 3) que permitió un análisis e interpretación de los resultados. 
Tabla 1. Registro de las búsquedas.

\begin{tabular}{ccccc}
\hline $\begin{array}{c}\text { Base de datos en } \\
\text { la que se ha } \\
\text { buscado }\end{array}$ & $\begin{array}{c}\text { Fecha de la } \\
\text { búsqueda }\end{array}$ & $\begin{array}{c}\text { Estrategia de } \\
\text { búsqueda }\end{array}$ & $\begin{array}{c}\text { Número de } \\
\text { artículos } \\
\text { encontrados }\end{array}$ & $\begin{array}{c}\text { Porcentaje de } \\
\text { artículos } \\
\text { relevantes }\end{array}$ \\
\hline $\begin{array}{c}\text { CUIDEN PLUS, } \\
\text { DOGPILE, }\end{array}$ & $\begin{array}{c}\text { De Junio a } \\
\text { PUBMED, LILACS, } \\
\text { ridec. }\end{array}$ & $\begin{array}{c}\text { Palabras Clave } \\
\text { Cuidado } \\
\text { profesional } \\
\text { Cuidado } \\
\text { humanizado }\end{array}$ & Cuarenta & $25 \%$ \\
\hline
\end{tabular}

Tabla 2 Síntesis de los estudios para generar una revisión de la literatura científica.

\begin{tabular}{|c|c|c|c|c|c|c|}
\hline \multicolumn{7}{|l|}{ Autor (es) } \\
\hline $\begin{array}{l}\text { Año y } \\
\text { Lugar } \\
\text { Titulo }\end{array}$ & $\begin{array}{c}\text { Propósito } \\
\text { Objetivo }\end{array}$ & Muestra & Medición & Tratamiento & $\begin{array}{l}\text { Resultados } \\
\text { cuantitativos }\end{array}$ & $\begin{array}{l}\text { Resultados } \\
\text { cualitativos }\end{array}$ \\
\hline ---- & -------- & --------------" & --------- & ------------- & |-------------. & ----------- \\
\hline
\end{tabular}

Las investigaciones están fundamentadas en las teorías de Enfermería Humanística de J. Phaterson, El cuidado Humanizado de J. Watson, y por el contexto hospitalario se ha considerado el Arte de la ayuda de la enfermería clínica de E. Wiedenbach, que a continuación se describe.

\section{EL SIGNIFICADO DEL CUIDADO DE ENFERMERÍA}

La palabra cuidado procede del latín "COGITATUM" que significa pensamiento y es definido a su vez como solicitud y atención con esmero para hacer bien alguna cosa.

Segundo Roach (1993) citado por Vera considera que el cuidado humano es una característica humana, es una forma de expresión, de relacionamiento con el otro ser, con el mundo, es una forma de vivir plenamente. Se inicia o se expresa de dos formas: como un modo de sobrevivir, y una expresión de interés y cariño se hace notar en todas las especies y sexos, ocurre entre los humanos predominantemente, considerando su raciocinio, capacidad de usar el lenguaje $u$ otras formas de comunicación.

Donahue (1985) citado por Vera establece la relación Enfermera/ Mujer/ Madre/ Cuidado. Ella considera que el papel de la enfermera es de madre, cuidadora, educadora; el cuidado comprende un sentido de afecto y preocupación, así como la responsabilidad por las personas necesitadas. El cuidado nace de un interés, de una responsabilidad, de una preocupación, de un afecto en el que implícitamente incluyen la maternidad y la educación, que a su vez implican ayudar a crecer.

El cuidar estuvo siempre presente con la historia humana. El cuidado como una forma de vivir, de relacionarse, el cuidado tecnológico también está presente en diversas civilizaciones, el cuidar fue parte de la medicina, por lo que nace la enfermería, comienza a tomar forma o adquirir realmente el status de una ocupación distinta; con la formalización de la enseñanza realizada por Florence Nightingale en 1960. Anteriormente la enfermería se desempeñaba a través de entrenamientos de personas para realizar actividades de confort de administración de medicamentos, de limpieza de unidad, cocina y lavanderías. Debido a la formación profesional se reconoce la enfermería como más bella que la medicina por la calidad de cuidado. El cuidado es una característica de enfermería que se intenta rescatar tanto en su conocimiento como en su arte (2).

Pinto (2001) presenta la siguiente definición: "cuidar es la esencia de enfermería, el cuidado es el elemento paradigmático que hace la diferencia entre enfermería y otras 
disciplinas del área de la salud". Las acciones de cuidar pueden ser observaciones positivas de soporte, comunicación, intervenciones físicas de la enfermería. Cuidar es dedicarse a un fin, a la protección, la mejoría y la preservación de la dignidad del otro. El profesional de enfermería tiene un reto grandioso: el lograr la humanización del cuidado en las unidades intensivas, considerando el conocimiento, la tecnología y los avances científicos bajo la premisa de respeto a la vida y la dignidad humana (3).

Strickland, citado por Morse, destaca que el cuidado es la fuente de nuestra conciencia y que al ser proporcionado se manifiesta como la expresión de nuestra humanidad y reflexión de nuestros valores, principios profesionales y personales. Considerando estas características, se puede describir el cuidado profesional como un rasgo humano, como un imperativo moral, como afecto, como un proceso de interacción personal y como intervención terapéutica (4). El cuidado que identifica a enfermería es el cuidado recíproco, interactivo e integrativo, en él las experiencias vividas y trascendentes contemplan la interacción entre personas totales con sus sentimientos, pensamientos y expresiones. Las actitudes son producto de antecedentes familiares que influyen en la historia de vida de cada ser; por tal razón, el ser es único e irrepetible. Estas concepciones tienen su referente teórico en el existencialismo cuyo exponente es el filósofo alemán Martin Heidegger, quien sostiene que el cuidado es una "condición existencial" y "una forma de ser"; por lo tanto, cada enfermera (0) es única (0) y trascendente en el proceso de cuidar y permite que la vida continúe, se desarrolle y se luche contra la muerte del individuo del grupo y de la especie, este es el modo de ser de la enfermera según Simona Roach. Lo descrito considera aspectos fundamentales que se deben considerar cuando nos encontramos en el proceso interactivo del cuidado, además la dimensión estética y ética del cuidado. La primera está conformada por los sentimientos y valores que fundamentan una acción en un contexto interrelacional de manera que exista coherencia y armonía entre el sentir, el pensar y el hacer; la segunda describe la necesidad de considerar el cuidar como un valor profesional y personal, siendo importante cumplir los patrones normativos de la profesión, que gobiernan las actitudes cuando se cuida al paciente, de allí que el cuidado como una práctica ética es la esencia de enfermería, evidenciado por el respeto a los clientes, familiares, a sí misma, a sus colegas de enfermería los miembros del equipo de salud y demás integrantes del sistema de servicios de salud (5).

\section{FUNDAMENTO FILOSOFICO DEL CUIDADO ENFERMERIA PROFESIONAL DE}

Watson (1985) citada por Marriner, sustenta su teoría sobre el ejercicio profesional de enfermería. En diez elementos básicos con componente fenomenológico dinámico ella sostiene que el hombre construye su propia historia, a través de experiencias sensibles con el otro ser. Esto es 1. Formación de un sistema de valores Humanístico-Altruista, que se aprenden al principio de la vida y resultan influidos en gran medida por los formadores de enfermería. 2. Infundir fe y esperanza, incorpora valores humanísticos, que facilita la promoción de un sistema de enfermería holística y potencia la salud entre los pacientes. 3. Cultivo de la sensibilidad hacia uno mismo y hacia los demás, identificación de sentimientos, donde la auto actualización de uno mismo mediante auto aceptación de la enfermera y el paciente, las hace más genuinas, auténticas y sensibles hacia los demás. 4. Desarrollo de una relación de ayuda-confianza, fundamental para la asistencia transpersonal. 5. Promoción y aceptación de la expresión de sentimientos positivos y negativos, debe reconocer la comprensión intelectual y la comprensión emocional. 6. Utilización sistemática del método de resolución de problemas para la toma de decisiones, el proceso de enfermería 
es similar al de investigación y sustenta el trabajo científico del profesional de enfermería. 7. Promoción de la enseñanzaaprendizaje interpersonal, factor importante de la enfermería, separa la asistencia de la curación, se fomenta el auto cuidado a través del proceso con técnicas de enseñanzaaprendizaje al paciente. 8. Provisión de un entorno de apoyo, protección y/o corrección mental, física, socio cultural y espiritual, reconocer la influencia de factores externos e internos en la salud-enfermedad de los individuos. 9. Asistencia con satisfacción de necesidades humanas, la enfermera reconoce las necesidades biofísicas, psicofísicas, psicosociales e interpersonales de ella misma y del paciente. 10. Tolerancia con las fuerzas fenomenológicas, describir los datos de la situación inmediata que ayuda a comprender los fenómenos en cuestión a través de la psicología existencial, según Watson difícil de entender, propone una experiencia motivadora del pensamiento que conduzca a una mejor comprensión. La responsabilidad de enfermería es enseñar métodos de resolución de problemas identificando la capacidad de afrontamiento y la adaptación a las pérdidas (6).

\section{LA ENFERMERÍA HUMANÍSTICA}

Paterson y Ziderad (1960) sustentan la teoría de la práctica humanística de enfermería como una experiencia existencial (interacción enfermera- paciente), reflexionar en ella, describir fenomenológicamente, los estímulos que se reciben, las respuestas, y lo que se llega a conocer a través de su presencia en el cuidado al prójimo, esto construirá la ciencia de la enfermería. La enfermería humanística considera una relación transaccional que se responsabiliza de investigar y cuya expresión demanda la conceptuación basada en la conciencia existencial que la/el enfermera (o) tiene de su ser y del otro ser y está relacionada con la conciencia intelectual; implicadas en ella las respuestas auditivas, olfatorias, orales, visuales, táctiles, cinestésicas y viscerales, y cada una de éstas pueden conferirle un significado único a la conciencia del hombre. Por lo que se precisa reflexionar, explorar, cuestionar las experiencias en el campo de enfermería, cuando se labora en los servicios hospitalarios, demostrar evidencias científicas considerando el existencialismo y la fenomenología, que sustentan la filosofía del cuidado de enfermería (7).

Además, considera que la enfermería humanística no es una cuestión de hacer sino de ser enfermera sensible a la realidad de la situación, entregada con todo su ser a la interacción enfermera-paciente. Esto implica una confirmación de la transacción ínter subjetiva con todas las capacidades humanas de uno que incluyen una respuesta a la realidad experimentada. La situación humana es el terreno en el que la enfermería toma forma y como tal, proporciona un marco de referencia en el cual ubicar el estudio y desarrollo de la enfermería humanística. Desde un punto de vista angular, la enfermería humanística enfoca la atención en la pregunta básica que subyace a la enfermería práctica. ¿Es humanizante o deshumanizante este evento ínter subjetivo particular y transaccional de la enfermería? Corresponde a las enfermeras asistenciales responder ésta interrogante.

\section{LA ENFERMERÍA HUMANÍSTICA COMO ARTE CLÍNICO}

Desde el punto de vista existencial, el arte de enfermería es un diálogo vivo, un encuentro genuino. La enfermera reconoce al paciente como alguien distinto a ella y se vuelca en él como presencia plena y auténtica que abarca todo su ser, con potencialidades. En el diálogo genuino y vivo, la enfermera percibe en el paciente la posibilidad de bienestar o de incrementar su humanidad (de confort, salud, o crecimiento). Como el hermoso paisaje que inspira al pintor o poeta, esta posibilidad del paciente se dirige a la enfermera, un llamado que demanda reconocimiento y respuesta. La relación que permite que la enfermera 
(artista) perciba esa posibilidad es real ya que establece una influencia recíproca. Si ella entabla una relación genuina con el paciente (yo-tú) sus capacidades reales (para el cuidado, su destreza en el trabajo, la esperanza) hacen resaltar la posibilidad (de bienestar, de humanidad, de confort, de crecimiento) del mismo modo que la capacidad y destreza del pintor o poeta crean una pintura o un poema.

Como arte clínico implica que el paciente debe participar como sujeto activo para hacer real su posibilidad, el arte de enfermería puede describirse como transaccional. No solo la enfermera ve posibilidades en el paciente, sino que el paciente también las ve en la enfermera (por ejemplo la posibilidad de ayuda, de confort, de apoyo) y él responde para que esto se produzca. Esta es la cuna de la vida verdadera según BUBER antropólogo, filósofo y rabino austriaco.

Arte y ciencia tienen un objetivo correspondiente, son respuestas humanas al mundo cotidiano en el que vive el hombre. En términos existenciales cada uno es una forma de diálogo vivo entre el hombre y su situación humana. En enfermería se debe hablar de "arte -ciencia" como un conjunto sintético nuevo; el diálogo de la enfermería refleja orientaciones de arte y ciencia pues comprende los mundos subjetivo y objetivo del paciente y la enfermera. Ella vive la síntesis del arte y la ciencia en el acto de la enfermería, éste es un fenómeno más fácil de vivir que de describir. Sin embargo, si verdaderamente experimentamos la enfermería como una especie de "arteciencia" como diálogo subjetivo-objetivo, entonces la enfermería ofrece una vía única hacia el conocimiento humano y es responsabilidad de la enfermera intentar describirla y compartirla (ob. cit. 7).

Wiedenbach (1960) citada por Marriner en su teoría el Arte de la Ayuda de la Enfermería Clínica, sostiene que el arte es una acción individualizada que lleva acabo la enfermera en una "relación biunívoca con el paciente" y constituye la respuesta consciente de la enfermera a los aspectos concretos de la situación real del paciente. El arte de la enfermera clínica está dirigida hacia la consecución de cuatro objetivos principales: 1. Entender al paciente, su patología, situación y necesidades. 2. Incrementar las actitudes del paciente. 3. Mejorar su patología o situación dentro del marco del plan terapéutico asignado. 4. Prevenir la recidiva de su problema o el desarrollo de otro que pueda producir ansiedad, incapacidad o agotamiento. El arte de enfermería comprende tres actividades iniciales: Estímulo (conducta que representa el paciente), Pronóstico (expectativa sobre la evolución del paciente), Interpretación (comparación de lo que se percibe con lo que se prevé o espera). El arte de enfermería no está constituido por acciones racionales o reaccionarias, sino por acciones deliberadas, es decir, en la aplicación de principios de ayuda para lograr comprender lo que el paciente quiere expresar con su conducta (ob.cit.6).

\section{ELEMENTOS PARA EL CUIDAR Y CUIDADO EN PACIENTES HOSPITALIZADOS}

El punto más crucial de dudas acerca de la concepción equivocada sobre el cuidar/ cuidado como una función esencialmente técnica desvirtuó a la enfermería. El cuidado implica una relación interpersonal irrepetible. Para que una enfermera cuide a un paciente hospitalizado precisa de lo siguiente: 1. Paciencia -Atención -Cariño. Entender al paciente con voluntad, ser servicial y con gran profesionalismo. 2. La persona que cuida debe sentir placer. 3. En la situación de cuidado se requiere piedad, esperanza, intención de la cuidadora. 4. Para ser cuidado se necesita dedicación, obediencia, armonía, colaboración, dependencia y presteza. 5. En la enfermera debe haber comprensión, aceptación, expresión y colaboración. 6. En relación a los efectos del cuidado debe haber: calor humano, buenos resultados, gratificación y entendimiento. Estos 
elementos están implícitos en la relación del cuidado, pocas veces descrito de modo claro y práctico, que implica términos filosófico ideológico y existencial, que se hacen necesarios en el cuidado de enfermería, que determina el sello de calidad del profesional (ob.cit.2).

\section{EL PROCESO DE CUIDAR}

En enfermería el proceso de cuidar es interactivo entre cuidadora y ser cuidado, la primera cumple un papel activo, y el segundo un papel más pasivo en función de su situación, que puede o no contribuir en el cuidado. El proceso de cuidado es definido como el desenvolvimiento de acciones, actitudes y comportamientos con base científica, experiencia, intuición y pensamiento crítico realizado para con el ser humano en el sentido de promover, mantener y recuperar su dignidad y totalidad humana. Ese sentido engloba integridad y plenitud física, social, emocional, espiritual e intelectual. En las fases de vivir y morir constituye un proceso de transformación de ambos: cuidadora y ser cuidado. En el cuidado intervienen factores de influencia general: medio ambiente (físico, social y administrativo), momento de cuidado y tecnología, que constituyen la cultura organizacional (ob.cit. 2).

\section{EL SISTEMA DE ENFERMERÍA CLÍNICA EN EL CUIDADO}

Los límites o las fronteras de un servicio profesional ofrecen las pautas individuales para continuar el ejercicio de la profesión. Los límites profesionales están enmarcados por el código profesional y las leyes, los límites locales lo establecen el hospital, clínica, o el individuo que contrata los servicios de la enfermera, y los límites personales lo impone la propia enfermera.

El sistema de la enfermería clínica, según Wiedenbach, lo constituyen: La enfermera (con filosofía explícita: hay un paciente que necesita ayuda), persona (con potencialidades y responsabilidades), salud (relación enfermera-paciente, para conservarla), entorno (facilitar los esfuerzos del individuo para superar los obstáculos que habitualmente interfieren en su capacidad para responder adecuadamente a lo que exige su condición, entorno, situación y época). Además identifica cinco atributos esenciales de un profesional:

1.Objetivo definido.

2. Maestría y conocimientos esenciales para la consecución del objetivo.

3. Capacidad para establecer y mantener relaciones de trabajos adecuados con otros individuos tanto profesionales como no profesionales e intereses por crear nuevos conocimientos. 5. Dedicación al logro de metas de la humanidad en vez de perseguir su gloria personal (ob.cit.5).

\section{Tabla 3 COMPARACIÓN Y CONTRASTE DE HALLAZGOS DE LOS ESTUDIOS DE CUIDADO HUMANIZADO EN ENFERMERÍA}

\begin{tabular}{|c|c|c|c|c|}
\hline $\begin{array}{c}\text { Autor(es) año, lugar y } \\
\text { título. }\end{array}$ & Hallazgo 1 & Hallazgo 2 & Hallazgo 3 & Hallazgo 4 \\
\hline $\begin{array}{l}\text { 1. Zarate Flores, Melani } \\
\text { Katalin } 2013 \text { Perú } \\
\text { "Influencia del cuidado } \\
\text { humanizado enfermero en } \\
\text { el afrontamiento de estrés } \\
\text { traumático en pacientes } \\
\text { que acuden al servicio de } \\
\text { emergencia del hospital } \\
\text { Félix Mayorca Soto" }\end{array}$ & $\begin{array}{l}\text { El } 78 \% \text { de } \\
\text { enfermeros brinda un } \\
\text { cuidado humanizado } \\
\text { eficaz. }\end{array}$ & $\begin{array}{l}\text { Los factores } \\
\text { relacionados a } \\
\text { brindar un cuidado } \\
\text { humanizado son } \\
\text { edad, sexo, tiempo } \\
\text { de servicio y } \\
\text { especialidad. }\end{array}$ & $\begin{array}{l}\text { Los pacientes } \\
\text { presentaron un nivel } \\
\text { de estrés leve } 86.7 \\
\% \text {. }\end{array}$ & $\begin{array}{l}\text { El afrontamiento de } \\
\text { estrés está } \\
\text { relacionado con el } \\
\text { cuidado } \\
\text { humanizado } \\
\text { recibido por el } \\
\text { personal } \\
\text { enfermería. }\end{array}$ \\
\hline
\end{tabular}


2. Colome Beck, Carmen. et.al. 2009, Colombia. "Humanización de asistencia de enfemería:Percepción de los enfermeros en los servicios de salud de un municipio"

3. Poblete Troncoso Margarita et. al. . 2007 Chile

"Cuidado humanizado: Un desafío para los enfermeros en los servicios hospitalarios"
Cuida al otro como a tí te gustaría que fueras cuidado.

(1)

(a)

Se

Tener una visión Falta de tiempo de integral de los los trabajadores usuarios. para brindar un cuidado humanizado

\begin{abstract}
4. Rivera Álvarez, L. et. al. 2005 Colombia.

"Percepciones comportamientos

de de cuidado humanizado de enfermería en la clínica del country" personas humanizado de enfermería.
\end{abstract}

Se observa en Las instituciones Latinoamérica la necesidad de sistemas complejos, buscar el significado con pautas y de cuidado en la normas enfermera, y que éste sea un "cuidado humanizado" y se incorpore a la praxis.

establecidas, están determinando que el acto de cuidar no sea centrado en el usuario, los pacientes valoran más la relación transpersonal.

El $86.7 \%$ de las El $12.04 \%$ de las personas casi siempre percibieron un cuidado humanizado de enfermería.
Es difícil mantener
los valores humanitarios en el acto de cuidar en las instituciones

públicas de salud en donde parecen invisibilizarse los cuidados de enfermería por la labores biomédicas.

El $1.09 \%$ de las personas

hospitalizadas en la Clínica del country solo algunas veces percibieron un cuidado humanizado
La estructura física inadecuada $\quad y$ carencia de recursos humanos y materiales no permiten brindar un cuidado humanizado.

El cuidado humanizado se percibió con comportamientos donde se priorizo a la persona $89.4 \%$, se destacaron características de la enfermera de empatía y pro actividad $89.8 \% \quad y$ se atendieron sentimientos del paciente $87.6 \%$.
5. Figueroa Guerra Evelia 2005 Venezuela

"El cuidado humano y la práctica de enfermería"

\section{Montilla Reyes, Nancy 2005 Venezuela}

" Significado que le asignan las enfermeras al cuidado humano del recién nacido prétermino"

7. Zambrano Amarilis 2003 Venezuela

"El cuidar de sí como valor de enfermería"

\section{La} etnográfica considera que debe existir cuidado humano, valores y creencias en la asistencia de enfermería.

\section{Categoría}

Diferenciando

cuidado humano.

\author{
1: Categoría 2: \\ el Condiciones \\ ambientales para el \\ cuidado humano
}

La cultura organizacional es significativa para el cuidado humano.

\section{Categoría \\ Comunicación cuidado humano}

3: Categoría 4:

y Actitud de la enfermera hacia el cuidado humano.
Cuidar de si es una responsabilidad

personal por

construcción humana e individual
La enfermera para cuidar de si a de fundamentarse en su propia escala de valores:

conocimiento de sí misma, autoestima, responsabilidad, madurez personal, respeto y dignidad.

8. Escobar Digna 2003 Las enfermeras
Venezuela
"El poder del cuidado
desconocen del
humano en la enfermera práctica del cuidado
desde una perspectiva humano.
fenomenológica"

La práctica del cuidado humano de enfermería está influenciado por el paradigma tecno científico

\section{Mantener el} equilibrio dinámico entre cuidar de si y cuidar de otros.

El cuidado humano conlleva a la trascendencia de la enfermera (o) por consiguiente el reconocimiento social de la 


\section{Arenas Nelly 2003 Venezuela \\ "La enfermera en la cotidianidad del cuidado humano"}

$\begin{array}{llr}\text { Categoría central: } & \text { La práctica diaria } \\ \text { Cuidado construcción } & \text { radica en el } \\ \text { del pensar ontológico } & \text { quehacer de la } \\ & \text { enfermera (o) bajo } \\ & \text { la cotidianidad de la } \\ & \text { relación enfermera- } \\ & \text { paciente y el } \\ & \text { entorno. }\end{array}$

10. Vila, Vanesa da Silva
Carvalho et.al. 2002 Brasil
"Significado cultural del
cuidado humanizado en la
unidad de terapia
intensiva"

Categoría 1:
Cuidado
Humanizado es
Amar al
prójimo como
así mismo.

profesión en el país.

Connotación

relevante de las

condiciones

intervinientes de

cada persona, las

cuales se forman

desde el momento

del ser y juega un

papel fundamental

para brindar el

cuidado como

persona humana a

quien lo necesita, lo

cual permitirá lograr

la reconstrucción

misma de esa

condición humana

en el ser cuidador

que presta sus

servicios.

Categoría 2:
Cuidado
humanizado no está
presente como
debiera estar, hay
estrés y sufrimiento,
es necesario cuidar
de quien cuida.

El tema cultural:

Cuidado

humanizado "mucho

se habla poco se

vive" de quien cuida.

\section{DISCUSIÓN}

El abordaje teórico del cuidado humanístico en enfermería y las evidencias de investigaciones realizadas en Latinoamérica relacionados al cuidado profesional demuestran que los factores sociales (edad, sexo, tiempo de servicio) y profesionales (especialidad) influyen en el cuidado humanizado y éste ayuda al afrontamiento del estrés al paciente durante la enfermedad en el servicio de emergencia (11). El cuidado humanizado es percibido con comportamientos donde se prioriza a la persona (89.4\%) con características de empatía y pro actividad de la enfermera $(89.8 \%)$ y atención del sentimiento del paciente (87.6\%) (14). Al respecto Roach en (1993) sustenta que el cuidado humanizado es una forma de expresión y relacionamiento con el otro ser. Donahue en (1985) refiere que el cuidado nace de un interés, responsabilidad de una preocupación y afecto que implican ayudar a crecer a la cuidadora y al ser cuidado es decir enfermera y paciente, esto es el cuidado que identifica a enfermería y debe ser recíproco interactivo e integrativo en el que se contemplan sentimientos, pensamientos y expresiones (5).

Las instituciones hospitalarias en Latinoamérica con sistemas complejos con pautas y normas establecidas están determinando que el acto de cuidar no esté centrado en el usuario, es difícil mantener los valores humanitarios en el acto de cuidar, los cuidados de enfermería parecen invisibilizarse por la labores biomédicas, considerar que, los pacientes valoran más la relación transpersonal (13). La estructura física inadecuada la carencia de recursos humanos y materiales la falta de tiempo de los trabajadores no permiten tener una visión integral de los usuarios (12). Esto limita el relacionamiento profesional para explorar sus vivencias durante la enfermedad y poder cuidarlo. La teorista Watson manifiesta que ante la deshumanización en el cuidado a causa de la reestructuración administrativa en la mayoría de sistemas de salud en el mundo 
es necesario rescatar el aspecto humano, espiritual, y transpersonal en la clínica, administrativa, investigativa y educativa por parte de los profesionales de enfermería (6), esto es una oportunidad para valorar y demostrar el rol de la enfermería en la sociedad.

La cultura organizacional es significativa para el cuidado humano, la calidad de la actividad profesional los valores y creencias fortalecen la humanización del cuidado (15). Estas consideraciones concuerdan con lo referido por Vera (2) ella sostiene que en el cuidado intervienen factores de influencia general, medio ambiente (físico, social y administrativo). Se debe diferenciar el cuidado profesional considerando las condiciones ambientales la comunicación y la actitud de la enfermera es fundamental (16). Según la teorista Paterson en el cuidado humanístico debe existir una relación existencial (enfermera-paciente), transaccional (interactuando en el cuidado) y una conciencia intelectual (atención a las respuestas humanas) considerando el tiempo y el espacio, permitir un encuentro genuino y un dialogo vivo, esto permitirá el ejercicio de la ciencia y arte de enfermería.

El cuidado como construcción del pensar ontológico, considera características universales propias de los seres humanos importantes en la vida cotidiana, esto exige que en la práctica diaria debe haber relación entre enfermera-paciente y el entorno, considerar que las condiciones intervinientes que se han ido formando en cada persona juega un papel fundamental para brindar el cuidado como persona humana, esto permite reconstruir la condición humana en el ser cuidador que presta sus servicios (19). El cuidar de sí es una responsabilidad personal por construcción humana e individual fundamentado en los valores de autoestima, responsabilidad, madurez, respeto y dignidad propia para mantener el equilibrio dinámico entre el cuidar de sí y cuidar de otros (17). Estas consideraciones permiten un crecimiento personal y profesional (2).
Existe desconocimiento de la práctica del cuidado humanístico y la trascendencia y reconocimiento social a que ésta lleva, la enfermería está influenciada por el paradigma tecno científico (18). Humanizar el cuidado es amar al prójimo como así mismo, esto no se evidencia, hay estrés y sufrimiento de los profesionales de enfermería en los hospitales, es necesario cuidar de quien cuida, mucho se habla de cuidado humanizado y poco se vive. (20). Es importante considerar que el usuario puede olvidar lo que vio, lo que escucho, pero jamás olvidará sus experiencias y quien lo cuido durante su estadía en el hospital.

\section{CONSIDERACIONES FINALES}

Los hallazgos de las investigaciones reportadas de Latinoamérica a la comunidad científica de enfermería de cuidar/ cuidado humanizado en los servicios hospitalarios evidencian que hay factores sociales $y$ profesionales que influyen en el cuidado humanizado.

Las instituciones hospitalarias con sistemas complejos, están determinando que el acto de cuidar no este centrado en el usuario, es difícil mantener los valores humanitarios por la labores biomédicas, la estructura física inadecuada, carencia de recursos humanos y materiales, falta de tiempo de los trabajadores, no permiten tener una visión integral del usuario.

La cultura organizacional y la calidad de la actividad profesional es significativa para el cuidado humanizado.

El cuidado como construcción del pensar ontológico, considera características propias de los seres humanos, la relación y comunicación entre enfermera-paciente y el entorno.

Existe desconocimiento de la práctica del cuidado humanístico y la trascendencia que esto implica, la enfermería está influenciada por el paradigma tecno científico. Es necesario asimilar los componentes filosóficos del cuidado y practicarla para encontrar una unidad de pensamiento y sustentar científicamente el cuidado 
profesional, esto permitirá la identidad y reconocimiento de la sociedad que exige un cuidado con dignidad humana.

\section{Correspondencia:}

Mg. Nelly Hinostroza Robles

Correo electrónico:

nmhinostroza@outlook.com

\section{REFERENCIAS BIBLIOGRAFICAS:}

1. Colegio de Enfermeros del Perú. CEP. Codigo de Etica y Deontología. LimaPerú 2005 Pág. 19

2. Vera $R$. Cuidado humano o resgate necesario. 1ra Edición. Porto Alegre, Brazil. Zagra Luzzatto. 1,998 P. 17-51149.

3. Pinto N.. Indicadores de cuidado en Enfermería. Actualización en Enfermería. Bogotá 2001. [Internet] Disponible: URL http//Colombia.com/ medicina/enfermeríacom/medicina/enfer mería4101.indicadores.

4. Morse J. "Conceptos of Caring and Caring as a concept" Advances in Nursing Science 1990 V.13 n.1 p.1-14.

5. Sánchez B. et al. Dimensiones del cuidado. Facultad de enfermería Universidad Nacional de Colombia 1998. p. 9.

6. Marriner T. Modelos y teorías de enfermería. 5ta Edición. Impreso en España 2008 p.88, 149

7. Paterson, J. Enfermería Humanística. Edit. Limusa México. 1ra reimpresión 1990 p. 20, 27, 149.

8. Luiris T. Los paradigmas como base del pensamiento actual en la profesión de enfermería. Rev. Cubana de Educ. Med. Sup. 2002 Citado 5-3-2011. [Internet] Disponible en: URL club.ensayos. com/paradigmas enfermería/244html.

9. Burns N. Investigación en enfermería. Desarrollo de la práctica enfermera basada en evidencia. 5ta Edición Elsevier España, S.L. 2012 Pág. 218230
10. Colegio de Enfermeros del Perú (CEP) Historia de la Enfermería Peruana.1ra Edición. Lima, Perú. 2011. p. 103

11. Zarate M. Influencia del cuidado humanizado enfermero en el afrontamiento del estrés traumático en pacientes del servicio de emergencia del Hospital Félix Mayorca Soto. Perú [Tesis de Lic. en Enf.] UNDAC Perú 2013

12. Colome C. et. al. Humanización de la asistencia de Enfermería, percepción de enfermeros en servicios de salud de un Municipio. Rev. Gaúcha Enf. Porto Alegre Brasil 2009 marzo. 30 (1): 60

13. Poblete T. Cuidado humanizado: Un desafío para las enfermeras en los servicios Hospitalarios. [Tesis de Dr. en Enf.] Universidad Concepción Chile 2007.

14. Rivera L. Percepciones de comportamientos de cuidado humanizado de enfermería en la clínica del country. Rev. av. enferm. xxv (1) Brasil 2005.

15. Figueroa E. El cuidado humano y la práctica de enfermería. [Tesis Dr. en Enf.] Rev. Latindex (4):4 Venezuela 2005.

16. Montilla N. Significado que le asignan las enfermeras al cuidado humano del recién nacido pre término críticamente enfermo. [Tesis Dr. en Enf.] Rev. Latindex (4):3 Venezuela 2005.

17. Zambrano A. El cuidar de si como valor de enfermería. [Tesis Dr. en Enf.] Universidad Carabobo. Venezuela 2003.

18. Escobar D. El poder del cuidado humano en la enfermera desde una perspectiva Fenomenológica. [Tesis Dr. en Enf.] Rev. Med. Book. Venezuela 2003 (4):2

19. Arenas N. La enfermera en la cotidianidad del cuidado humano. [Tesis Dr. en Enf.] Rev. med. Book. Venezuela 2003 (4): 3 
20. Vila V. Significado cultural del cuidado humanizado en la unidad de terapia intensiva. [Tesis Dr. en Enf.] Universidad Sao Paulo Brasil 2002.

Recibido: 20/01/14

Aprobado: 25/05/14 